\title{
Propriedades Psicométricas da Versão Brasileira da Escala de Comprometimento com a Carreira
}

Psychometric Properties Of The Brazilian Version

Of The Career Commitment Scale

Propiedades Psicométricas De La Versión Brasileña

De La Escala De Compromiso Con La Carrera

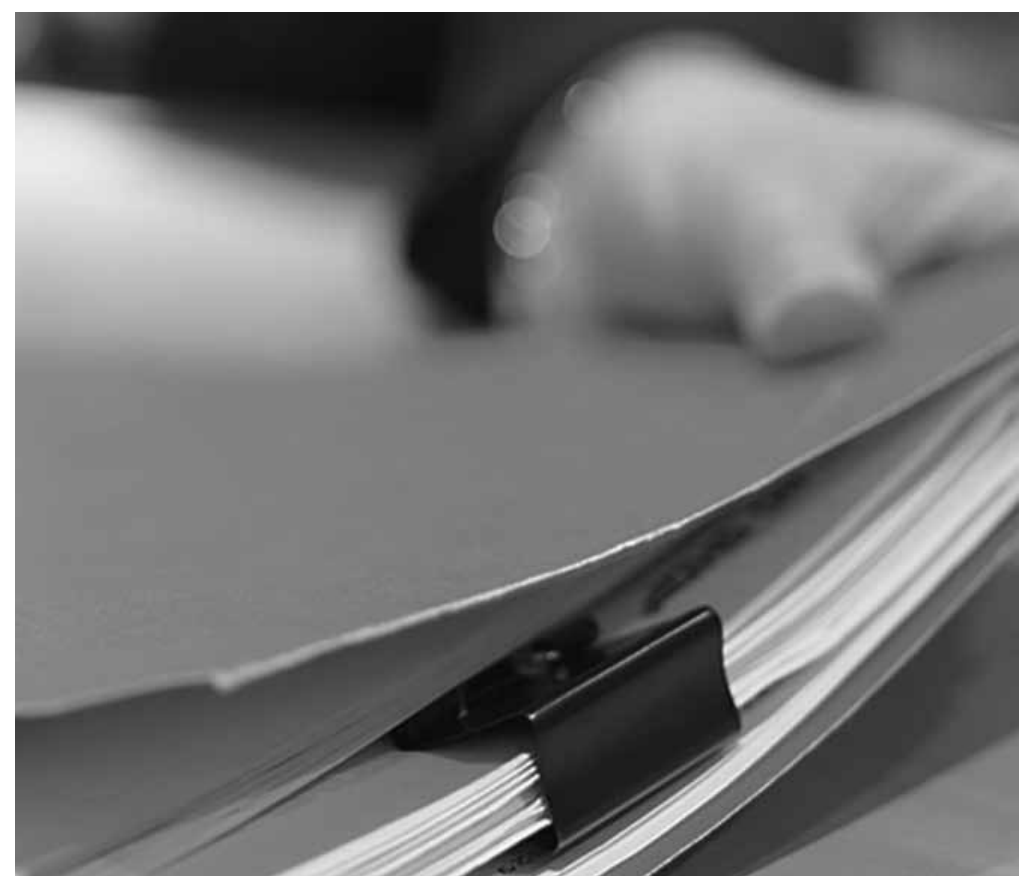


Resumo: A definição e a mensuração do vínculo do trabalhador com sua carreira adquirem relevância no contexto de instabilidade que caracteriza o mundo do trabalho contemporâneo. O comprometimento com a carreira significa a motivação e a efetiva dedicação do indivíduo para desenvolver-se como trabalhador em determinado campo de atividade. Foram investigadas, através de análise fatorial e de consistência interna, as características psicométricas de uma versão brasileira da Escala de Comprometimento com a Carreira (ECC) (Carson \& Bedeian, 1994). A ECC é composta por três fatores: identidade, planejamento e resiliência. Uma amostra de 668 trabalhadores, 415 homens e 253 mulheres, com idades entre 25 e 65 anos, com tempo de carreira mínimo de 5 anos, níveis de escolaridade entre ensino técnico e pósgraduação, respondeu a escala. A análise fatorial encontrou os três fatores correspondentes às dimensões propostas para o construto. Os indicadores de consistência interna obtidos para a escala total e para cada dimensão de comprometimento foram satisfatórios, revelando que todos os itens contribuem para aumentar a precisão do instrumento. Níveis de escolaridade mais elevados foram associados a maior identidade de carreira, e as análises indicam qualidades de validade de construto e de precisão satisfatórias para a versão brasileira da Escala de Comprometimento com a Carreira.

Palavras-chave: Comprometimento. Desenvolvimento profissional. Motivação. Identidade profissional.

Abstract: The definition and assessment of the worker's connection with his/her career is relevant in the context of instability which characterizes the contemporary labor world. Career commitment means the individual's motivation to dedicate to his/her development as a worker in a determined field of activity. The psychometric properties of the Brazilian version of the Career Commitment Scale (CCS) were investigated. The CCS is composed of three factors: identity, planning and resilience. A sample of 668 workers, 415 men and 253 women, ages ranging between 25 and 65 years old, with a minimum of five years of career time and levels of education between technical training and post-graduation answered the scale. The factorial analysis found three factors according to the dimensions proposed for the construct. Internal consistence indicators were satisfactory both for the total scale and each entrenchment dimensions, revealing that all items contribute for higher scale reliability. Higher levels of education showed association with enhanced career identity. The analysis showed satisfactory construct validity and reliability qualities for the Brazilian version of the Career Commitment Scale.

Keywords: Commitment. Professional development. Motivation. Professional identity.

Resumen: La definición y la mensuración del vínculo del trabajador con su carrera adquieren relevancia en el contexto de inestabilidad que caracteriza el mundo del trabajo contemporáneo. El compromiso con la carrera significa la motivación y la efectiva dedicación del individuo para desarrollarse como trabajador en determinado campo de actividad. Fueron investigadas, a través de análisis factorial y de consistencia interna, las características psicométricas de una versión brasileña de la Escala de Compromiso con la Carrera (ECC) (Carson \& Bedeian, 1994). La ECC está compuesta de tres factores: identidad, planificación y resiliencia. Una muestra de 668 trabajadores, 415 hombres y 253 mujeres, con edades entre 25 y 65 años, con tiempo de carrera mínimo de 5 años, niveles de escolaridad entre enseñanza técnica y posgrado, contestó la escala. El análisis factorial encontró los tres factores correspondientes a las dimensiones propuestas para el constructo. Los índices de consistencia interna obtenidos para la escala total y para cada dimensión de compromiso fueron satisfactorios, revelando que todos los ítems contribuyen para aumentar la precisión del instrumento. Niveles de escolaridad más elevados fueron asociados a mayor identidad de carrera, y los análisis indican calidades de validez de constructo y de precisión satisfactorias para la versión brasileña de la Escala de Compromiso con la Carrera.

Palabras-clave: Compromiso. Desarrollo profesional. Motivación. Identidad profesional.

As mudanças no cenário do mundo do trabalho nas últimas décadas transformaram o significado das carreiras profissionais. Em tempos passados, ter uma carreira significava estabilidade, uma trajetória previsível de promoções, e a aposentadoria. Na atualidade, o trabalho é, cada vez mais, uma relação sem vínculos de longo prazo. Desse modo, as carreiras tornaram-se mais centradas no indivíduo do que em relações institucionais
(Sullivan \& Baruch, 2009). Nesse contexto, questões sobre a definição e a mensuração do vínculo do trabalhador com sua carreira adquirem relevância. Este artigo apresenta as qualidades psicométricas da versão brasileira da Escala de Comprometimento com a Carreira desenvolvida por Carson e Bedeian (1994). Primeiramente, será esclarecido o conceito de carreira e problematizado o significado de comprometimento com a 
De acordo com Blau, o comprometimento com a carreira é uma atitude relacionada a um sentimento positivo do indivíduo em relação "à própria vocação, incluindo a profissão" (1988, p.295). carreira na atualidade, a fim de contextualizar e apresentar a proposta de operacionalização desse construto por Carson e Bedeian, e também de esclarecer a pertinência desse modelo para medir o comprometimento nas carreiras contemporâneas.

\section{O conceito de carreira}

Existe confusão no uso dos termos profissão, carreira e ocupação. Para Bastos, Magalhães e Carvalho (2010), ocupação refere-se ao conjunto de atividades necessárias para produzir um bem ou serviço. O termo profissão agrega, como dimensão descritiva, o grau de profissionalismo das ocupações. Uma ocupação assume o status de profissão na medida em que requer um longo processo de formação e de treinamento, legitimado socialmente como fundamento para o exercício de autoridade em relação aos temas de sua competência, que possui um código de ética regulador e uma cultura profissional estável. Por fim, o termo carreira significa uma sequência de trabalhos articulados ao longo do tempo, que pode incluir ocupações e/ou profissões, agregando uma dimensão temporal. Nesse sentido, Grzeda (1999) considera que a natureza sequencial da noção de carreira implica a aquisição progressiva de comportamentos e de atitudes necessários ao desenvolvimento profissional.

Ainda sobre o entendimento do termo carreira, é importante citar o aporte de Schein (1990), a partir do trabalho de Hughes (1958), de duas perspectivas de significado: a externa e a interna. A carreira externa refere-se à história dos cargos, das responsabilidades e das funções exercidas pelo trabalhador, o que pode ser verificado por um observador externo. A carreira interna é a perspectiva subjetiva do indivíduo sobre as suas experiências de trabalho, os significados particulares que atribui a sua trajetória profissional. Em ambas as perspectivas, a carreira é definida como um encadeamento de atividades, de posições e de papéis de trabalho ao longo do tempo.

\section{Comprometimento com a carreira}

De acordo com Blau, o comprometimento com a carreira é uma atitude relacionada a um sentimento positivo do indivíduo em relação "à própria vocação, incluindo a profissão" (1988, p.295). Outros autores diferenciaram os aspectos atitudinal e comportamental do comprometimento (Bastos, 1997). O apego afetivo a uma profissão/ocupação seria um antecedente do engajamento efetivo do indivíduo aos comportamentos necessários ao progresso na carreira. Apesar da distinção analítica, esses dois aspectos se mostraram empiricamente relacionados (Goulet \& Singh, 2002). De acordo com Magalhães (2005), o comprometimento deve implicar não somente uma atitude favorável à permanência na ocupação ou profissão, mas, principalmente, o envolvimento do indivíduo no seu desenvolvimento profissional. Essa manifestação comportamental do comprometimento tem sido descrita como o estabelecimento de metas de carreira (Goulet \& Singh, 2002; Carless, 2005; Fu, 2011), o tempo gasto em investimentos na carreira (Bastos, 1997; Chang, 1999; Goulet \& Singh, 2002) e o enfrentamento de obstáculos de carreira (Bastos 1997; Carless, 2005; Fu, 2011). Meyer e Herscovitch (2001) observaram que as diversas definições do comprometimento com a carreira convergem ao descrever uma força estabilizadora que direciona o comportamento do indivíduo para atingir metas de carreira. De modo similar, Riveros e Tsai (2011) consideram que a presença de uma força motivacional direcionada ao alcance de metas em determinado papel de carreira é uma ideia central e recorrente nas definições atuais do construto. Por fim, Niu 
(2011) resumiu o conceito como a atitude do indivíduo em relação ao seu desenvolvimento profissional.

As diversas facetas do comprometimento com a carreira já estavam presentes no estudo de London (1983), um texto seminal nesse campo de estudo; o autor descreveu três componentes fundamentais do que denominou motivação de carreira, a saber: identidade, resiliência e planejamento. A identidade de carreira refere-se ao quanto o indivíduo define a si mesmo através do trabalho que realiza, o desejo de crescimento e de reconhecimento em seu campo de atividade. A resiliência refere-se as atitudes e comportamentos pertinentes à superação de circunstâncias problemáticas que venham atingir a carreira; desse modo, implica a autoconfiança para superar adversidades, a aceitação de desafios e de novos aprendizados e a disponibilidade para arriscar-se. E, por fim, o planejamento inclui o autoconhecimento do profissional com relação as suas forças e fraquezas, pois, a partir dessa autoavaliação, será capaz de estabelecer metas de carreira realistas e de planejar seu desenvolvimento.

Observa-se que o modelo de London já apresentava uma conjugação de aspectos atitudinais e comportamentais do comprometimento. Na verdade, são processos sobrepostos e de difícil distinção empírica. Em termos da operacionalização do constructo, fica claro que se devem considerar essas diferentes dimensões ou fatores.

\section{Operacionalização e mensuração do comprometimento com a carreira}

A mensuração do comprometimento com a carreira tem um histórico controverso devido à problemática definição do construto. Nas pesquisas sobre o tema, destaca-se o uso de duas medidas: a escala unidimensional de oito itens, desenvolvida por Blau (1985), e a escala de três fatores (correspondentes ao modelo de London, 1983) de doze itens, desenvolvida por Carson e Bedeian (1994).

Blau, baseado em sua concepção de carreira como uma vocação, definiu o comprometimento como "a atitude do indivíduo em relação a sua profissão ou vocação" (1985, p.278). O conteúdo dos oito itens propostos por Blau enfatiza a certeza do sujeito sobre a escolha de sua profissão, focando a intensidade do desejo de permanecer na atividade escolhida em vez de abandoná-la.

A medida de Blau já foi recomendada por ser possuidora de propriedades psicométricas adequadas (Morrow, 1993), mas, por outro lado, foi criticada quanto a sua validade de construto por Carson e Bedeian (1994). De acordo com a argumentação acima apresentada, esses autores observaram a forte sobreposição dessa medida com cognições de evasão de carreira, colocando em dúvida a validade de construto da medida de comprometimento de carreira de Blau. Entre os itens dessa escala, validada para o Brasil por Bastos (1992), estão "se eu pudesse escolher uma profissão diferente, eu escolheria", "se eu pudesse fazer tudo novamente, eu não escolheria trabalhar nesta profissão", e "esta minha profissão é ideal para trabalhar o resto da vida". Observa-se que esses e outros itens da escala correspondem a uma concepção de comprometimento entendido como o desejo de permanência na profissão ou ocupação.

A associação indevida entre comprometimento e intenções de permanência-evasão em uma ocupação/profissão é uma problemática também enfrentada pelos estudos e medidas 


\section{A obra clássica de D. Super mostrou que as vocações podem sofrer alterações na medida em que o autoconceito vocacional se modifica ao longo da vida (Super, Savickas, \& Super,} 1996). do comprometimento organizacional, que têm origem na preocupação com a rotatividade de trabalhadores. Em relação ao comprometimento organizacional, Rodrigues e Bastos esclarecem que "o 'ser comprometido' que, até o final do século XX, estava associado à ideia de permanência, passa a incorporar a noção de contribuição ativa para a organização" (2010, p.131). $\mathrm{E}$ as definições de comprometimento no trabalho têm sofrido intensa reformulação a fim de incorporar as dimensões relativas à efetiva identificação e aos investimentos do indivíduo nos diversos componentes da sua vida de trabalho, seja a carreira, seja a organização. Transpondo o raciocínio de Solinger, Olffen e Roe (2008) do âmbito organizacional para o foco carreira, é possível dizer que a permanência ou não do trabalhador em uma linha de trabalho/ carreira pode ser explicada por muitas variáveis além do comprometimento, e que a permanência, portanto, deve ser tratada apenas como uma possível consequência do comprometimento. Ora, trabalhadores muito comprometidos com suas carreiras podem fazer novas escolhas ocupacionais por várias razões (mudanças de valores e de prioridades de vida, circunstâncias familiares), e trabalhadores pouco comprometidos podem permanecer em suas ocupações por motivos financeiros ou por falta de outras oportunidades de trabalho.

Além disso, de acordo com Magalhães (2005), a convicção de uma escolha de carreira, e o entusiasmo decorrente, nem sempre resultam nos comportamentos necessários para superar os obstáculos que colocarão em teste a certeza e a força dessa escolha. Entre esses comportamentos, estão os aspectos de planejamento e de resiliência descritos por London, ausentes na escala de Blau. Essas limitações do instrumento estão logicamente relacionadas à definição do construto carreira na qual o autor se baseou. Blau (1985) considerou a carreira um equivalente de vocação, sem esclarecer mais precisamente quais eram esses dois conceitos e qual a relação entre eles. $\mathrm{O}$ autor parece ter ignorado o componente dinâmico desses conceitos, qual seja, vocações e carreiras estão em contínuo desenvolvimento. A obra clássica de D. Super mostrou que as vocações podem sofrer alterações na medida em que o autoconceito vocacional se modifica ao longo da vida (Super, Savickas, \& Super, 1996). O comprometimento com a carreira, portanto, pode resultar no desejo de permanência em uma profissão ou ocupação, mas somente o investimento pessoal em um processo contínuo de desenvolvimento profissional, tanto externa quanto internamente definido (Schein, 1990), justifica dizer que alguém possui uma carreira. Nesse sentido, reforçase a adequação do modelo de London, e também a necessidade de buscar melhor operacionalização do construto.

Em estudos posteriores, Blau (2003) propôs uma medida de comprometimento ocupacional de quatro dimensões, fazendo uma transposição do modelo de comprometimento organizacional de Meyer, Allen e Smith (1993). No entanto, esse modelo de vínculo com a organização tem sido profundamente revisto em sua validade, e a transposição feita por Blau traz os mesmos problemas para o estudo do vínculo com a carreira. A inconsistência central observada foi a inclusão de bases psicológicas instrumentais e afetivas de vínculo como partes do conceito de comprometimento, pois pesquisas recentes revelaram que a base instrumental não pode ser considerada fonte de comprometimento (Magalhães, 2008; Rodrigues \& Bastos, 2010).

Carson e Bedeian propuseram uma medida de comprometimento de carreira a partir da base conceitual oferecida por London. Desse modo, o comprometimento de carreira é tratado como um construto 
multidimensional com três componentes, descritos pelos autores da seguinte maneira: a identificação e o significado pessoal do trabalho (identidade), a autoavaliação de necessidades de desenvolvimento e o estabelecimento de metas de carreira (planejamento de carreira), e a resistência à ruptura da carreira em face de adversidades (resiliência). Essa medida aproxima-se das definições atuais do comprometimento, com significado de identificação e de envolvimento ativo na conquista de objetivos de carreira (ou da organização, no caso do comprometimento organizacional).

A medida desenvolvida por Carson e Bedeian é composta de doze itens e de três fatores com quatro itens cada. Os estudos de construção e validação resultaram em alphas de Cronbach entre 0.79 e 0.85 . Os 12 itens explicaram $64 \%$ da variância, e os autovalores foram 4,02 (Fator 1), 2,36 (Fator 2) e 1,38 (Fator 3). O primeiro fator, identidade de carreira, explicou 33,5\% da variância, com cargas fatoriais entre 0,57 e 0,80; o segundo, resiliência, explicou 19,7\%, com cargas fatoriais entre 0,63 e 0,83 , e o terceiro, planejamento, explicou $11,5 \%$ da variância, com cargas fatoriais entre 0,47 e 0,77 .

A versão brasileira da Escala de Comprometimento de Carreira de Carson e Bedeian foi utilizada em alguns estudos brasileiros (Baiocchi \& Magalhães, 2004; Magalhães \& Gomes, 2005, 2007; Magalhães, 2008; Rowe \& Bastos, 2010, 2011). Baiocchi e Magalhães examinaram a relação entre comprometimento e entrincheiramento na carreira, e encontraram correlação entre identidade de carreira e custos emocionais, uma dimensão do entrincheiramento que mede a percepção de sofrimento emocional associado a uma possível mudança de carreira. Esse resultado foi corroborado nos estudos de Magalhães e Gomes e confirmam achados anteriores de Carson, Carson e Bedeian (1995). A pesquisa de Magalhães e Gomes (2005) revelou que o comprometimento está positivamente relacionado a uma medida de generatividade. Essa relação confirmou expectativas teóricas, pois a generatividade está ligada ao desejo de contribuir para o melhoramento da vida humana e de ser lembrado na posteridade (Schnell, 2011), e o comprometimento com a carreira pode significar a motivação e o engajamento do indivíduo para contribuir socialmente através do papel de trabalhador.

Este estudo investigou as propriedades psicométricas da versão brasileira da Escala de Comprometimento de Carreira proposta por Carson e Bedeian. Os estudos brasileiros anteriormente realizados com o instrumento não relataram em detalhes suas características psicométricas e não apresentaram a totalidade dos itens que compõem a escala. O presente artigo vem oferecer aos pesquisadores do comportamento no trabalho uma descrição e uma análise mais pormenorizada dessa medida, a fim de ampliar a sua utilização no contexto brasileiro. Foram investigadas diferenças de comprometimento associadas à escolaridade, ao sexo e ao tipo de vínculo de trabalho. O exame da variável escolaridade foi considerado relevante tendo em vista a magnitude de investimentos e de processos de identificação, de resiliência e de planejamento envolvidos na aquisição de títulos acadêmicos, o que resulta na expectativa de que trabalhadores com escolaridade mais elevada se mostrem mais comprometidos. A investigação das diferenças relacionadas ao sexo se mostra pertinente considerando as distintas contextualizações social, cultural e econômica do papel de trabalho na vida de homens e mulheres. Por fim, foram comparadas variáveis de comprometimento com a carreira de (a) trabalhadores celetistas, ligados a organizações da iniciativa privada e com relações de 
trabalho reguladas pelas leis trabalhistas, e (b) trabalhadores do serviço público, ocupantes de cargos públicos providos por concurso público, com estabilidade e planos de carreira assegurados em regime estatutário. $\mathrm{O}$ tipo de contrato de trabalho pode resultar em maior ou menor preocupação com aspectos de planejamento de carreira, por exemplo. Espera-se que trabalhadores em contratos celetistas se mostrem mais preocupados com o planejamento de suas carreiras, uma vez que não estão amparados por uma estrutura de carreira estável e previsível, tal como no regime estatutário do setor publico, e que enfrentam a instabilidade do mundo do trabalho.

\section{Método}

\section{Participantes}

Participaram do estudo 668 trabalhadores das Regiões Sul e Nordeste do Brasil, situados em diversas áreas de atividade e setores da economia, sendo $62,13 \%$ homens e $37,87 \%$ mulheres, com idades entre 25 e 65 anos (média $=35,2$ ). Em termos de escolaridade, 236 possuíam ensino técnico, 275, ensino superior (completo ou em andamento) e 157, pós-graduação completa ou em andamento. Todos os participantes possuíam vínculo empregatício permanente e mais de cinco anos de carreira profissional, na mesma ou em diversas organizações de trabalho. Sobre o tipo de contrato de trabalho, $64,72 \%$ eram empregados de organizações da iniciativa privada contratados em regime celetista (CLT), e 35,28\% eram empregados de organizações do setor público vinculados por regime estatutário, considerando os 598 participantes que informaram esse dado. Os participantes responderam o instrumento por ocasião da coleta de dados para uma pesquisa mais extensa sobre o desenvolvimento de carreira na vida adulta.

\section{Instrumento}

Foi utilizada uma versão brasileira da Escala de Comprometimento na Carreira (ECC) desenvolvida por Carson e Bedeian (1994). A escala possui doze itens distribuídos em três fatores com quatro itens cada, a saber: identidade, resiliência e planejamento. Os itens são frases que descrevem atitudes e comportamentos em relação à carreira profissional, e as respostas são assinaladas em uma escala Likert de cinco pontos, indicando o grau de concordância com as frases. O instrumento original, em inglês, foi traduzido para o português, separadamente, por três psicólogos bilíngues. As versões foram comparadas e não mostraram diferenças dignas de nota. A tradução obtida foi revertida para o inglês por outros dois psicólogos bilíngues (back-translation) a fim de verificar sua equivalência semântica e sintática. Não foram necessários ajustamentos. O estudopiloto foi realizado com 32 trabalhadores. Entre eles, 15 estavam matriculados em cursos de pós-graduação nas áreas de Administração e Engenharia, e 17, em cursos de formação de tecnólogos em gestão de recursos humanos e de logística empresarial. O instrumento mostrou-se de fácil compreensão.

\section{Procedimentos}

A coleta de dados foi coletiva, realizada no local de trabalho ou de estudo dos participantes com prévio consentimento das autoridades responsáveis. A pesquisa foi realizada mediante aprovação do Comitê de Ética da instituição a que o pesquisador estava afiliado por ocasião da pesquisa, e foram atendidas as determinações éticas da Resolução no 196/1996, do Conselho Nacional de Saúde (CNS, 1996), sobre a pesquisa com seres humanos. 
Análise dos dados

A validação das dimensões de comprometimento foi realizada por medidas de adequação dos dados à análise fatorial, determinação do número de fatores adequados à análise fatorial e verificação da distribuição dos itens em dimensões pelo método de componentes principais. A precisão foi avaliada por meio do alfa de Cronbach, tendo sido utilizada ANOVA para comparar os escores de comprometimento com a carreira na dependência da escolaridade, sexo e tipo de contrato de trabalho.

\section{Resultados}

Antes de proceder ao cálculo de análise fatorial, a adequação da amostra foi verificada pelo determinante da matriz de correlação, pelo coeficiente de adequação da amostra de Kaiser-MeyerOlkin (KMO) e pelo Teste de Esfericidade de Bartlett. O coeficiente Kaiser-Meyer-Olkin (KMO $=0,877)$, o determinante da matriz de correlações $(0,036)$ e o Teste de Esfericidade de Bartlett $(\mathrm{c} 2[78, N=668]=2036,15 ; p<0,01)$ revelaram adequação satisfatória dos dados à análise fatorial (Dassa, 1999). Assumindo que as dimensões não são a priori correlacionadas, uma vez que remetem a aspectos distintos do processo de comprometimento, uma análise de componentes principais seguida por rotação Varimax foi utilizada para examinar a estrutura fatorial da ECC. O gráfico Scree indicou três fatores como a melhor explicação possível para os dados apresentados. Três fatores obtiveram autovalores adequados (eigenvalue $>1,000$ ) e explicaram mais da metade da variância dos escores de comprometimento (56,22\%). Os autovalores foram 3,74 (Fator 1), 1,76 (Fator 2) e 1,25 (Fator 3). O primeiro fator (ID - identidade de carreira) explicou 31,18\%, o segundo (PL - planejamento de carreira) explicou 14,64\%, e o terceiro (RE - resiliência), 10,40\% da variância (Tabela 1).

A análise fatorial revelou que apenas um item saturou acima de 0,30 (valor mínimo definido para carga fatorial significativa) em mais de um fator. Esse item foi mantido no fator com maior saturação, considerando que a coerência semântica foi atendida e também observando que a magnitude da diferença entre a maior e a menor saturação foi superior a 0,1 (valor mínimo definido para diferença significativa). Os três fatores encontrados agruparam os itens correspondentes às respectivas dimensões do instrumento original e, portanto, revelaram as coerências semânticas pertinentes (Tabela 1). 
Tabela 1. Matriz estrutural das dimensões de comprometimento na carreira

№ Descrição do item $\quad$ Dimensões

1 Minha linha de trabalho/campo de carreira é uma parte importante de quem eu sou.

ID PL RE

2 Minha linha de trabalho/campo de carreira tem um grande significado pessoal para mim.

3 Eu não me sinto emocionalmente apegado a esta linha de trabalho/campo de carreira. (inv)

4 Eu estou fortemente identificado com a linha de trabalho/

campo de carreira que escolhi.

0,751

5 Eu tenho uma estratégia para alcançar meus objetivos nesta

linha de trabalho/campo de carreira.

0,837

6 Eu criei um plano para meu desenvolvimento nesta linha

de trabalho/campo de carreira.

0,860

7 Eu tenho metas específicas para meu desenvolvimento nesta

linha de trabalho/campo de carreira.

8 Eu não costumo pensar sobre o meu desenvolvimento pessoal nesta linha de trabalho/campo de carreira. (inv)

$0,304 \quad 0,429$

9 Os desgastes associados à minha linha de trabalho/campo de carreira às vezes me parecem grandes demais. (inv)

10 Os problemas que eu encontro nesta linha de trabalho/campo de carreira às vezes me fazem questionar se os ganhos estão sendo compensadores. (inv)

11 Os problemas desta linha de trabalho/campo de carreira me fazem questionar se o fardo pessoal está valendo a pena. (inv)

$12 \mathrm{O}$ desconforto associado à minha linha de trabalho/campo de carreira às vezes me parece muito grande. (inv)

Eigenvalues

Porcentagem de variância explicada

Os índices de consistência interna (alfas de Crombach) obtidos para a escala total e para os escores parciais de cada dimensão de comprometimento foram os seguintes: 0,83 (ECC), 0,77 (ID - identidade de carreira), 0,75 ( $\mathrm{PL}$ - planejamento de carreira) e 0,72 (RE - resiliência). Foi verificada a contribuição de cada item para a precisão de sua dimensão. Os resultados obtidos mostraram que todos os itens contribuem para aumentar a precisão da sua respectiva dimensão (Tabela 2).

Tabela 2. Índices alpha de Cronbach por dimensão e se item deletado em cada dimensão da Escala de Comprometimento de Carreira

\section{Alpha se item deletado}

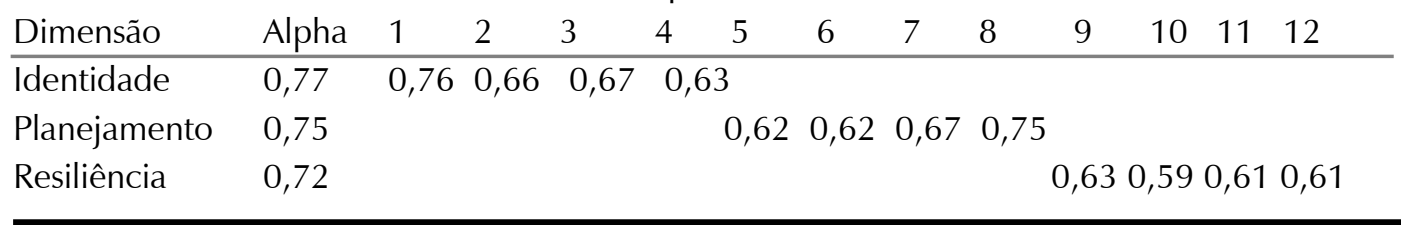


A análise de variância não revelou diferenças nos escores totais de comprometimento na dependência do nível de escolaridade, $\underline{\underline{F}}(2,666)=1,77, p=0,17$. Análises específicas para cada dimensão de comprometimento revelaram que não houve diferenças em resiliência, $\underline{F}(2$, $666)=1,69, p=018$, em planejamento de carreira, $\underline{E}(2,666)=2,25, p=0,10$, mas somente em identidade de carreira, $\underline{\mathrm{F}}(2,666)=3,213, p=0,04$, relacionadas ao nível de escolaridade. Trabalhadores com escolaridade em nível de pós-graduação apresentaram escores mais elevados do que aqueles com escolaridade de ensino técnico. A Tabela 3 apresenta dados de estatística descritiva dos escores de comprometimento em cada nível de escolaridade.

Tabela 3. Médias (M), desvios-padrão (dp) e valores de $\mathrm{N}$ para os escores de comprometimento com a carreira na dependência do nível de escolaridade

\begin{tabular}{llllll}
\hline Escolaridade & $\mathrm{N}$ & $\begin{array}{c}\text { Comprometimento } \\
\mathrm{M}(\mathrm{dp})\end{array}$ & $\begin{array}{c}\text { Identidade } \\
\mathrm{M}(\mathrm{dp})\end{array}$ & $\begin{array}{c}\text { Resiliência } \\
\mathrm{M}(\mathrm{dp})\end{array}$ & $\begin{array}{c}\text { Planejamento } \\
\mathrm{M}(\mathrm{dp})\end{array}$ \\
\hline Ensino técnico & 236 & $44,66(7,83)$ & $15,65(3,50)$ & $12,85(3,72)$ & $15,96(3,44)$ \\
Ensino superior & 275 & $44,82(8,83)$ & $16,41(3,58)$ & $13,11(3,93)$ & $15,35(3,72)$ \\
Pós-Graduação & 157 & $46,26(7,63)$ & $16,93(3,36)$ & $13,62(3,80)$ & $15,96(3,29)$ \\
Total & 668 & $45,06(8,22)$ & $16,27(3,52)$ & $13,12(3,83)$ & $315,71(3,53)$ \\
\hline
\end{tabular}

A análise de variância não revelou diferenças significativas entre os sexos no escore total de comprometimento, $\underline{\mathrm{F}}(1,667)=0,59, p=0,54$, e nos escores parciais de identidade, $\underline{\mathrm{F}}(1,667)$ $=0,74, p=0,39$, resiliência, $\underline{\mathrm{F}}(1,667)=0,15, p=0,69$, e planejamento, $\underline{\mathrm{F}}(1,667)=0,44$, $p=0,50$. A Tabela 4 apresenta dados de estatística descritiva dos escores de comprometimento em cada sexo.

Tabela 4. Médias (M), desvios-padrão (dp) e valores de $\mathrm{N}$ para os escores de comprometimento com a carreira na dependência do sexo

\begin{tabular}{|c|c|c|c|c|c|}
\hline Sexo & $\mathrm{N}$ & $\begin{array}{l}\text { Comprometimento } \\
\qquad \mathrm{M}(\mathrm{dp})\end{array}$ & $\begin{array}{l}\text { Identidade } \\
M(\mathrm{dp})\end{array}$ & $\begin{array}{l}\text { Resiliência } \\
M(\mathrm{dp})\end{array}$ & $\begin{array}{l}\text { Planejamento } \\
M(\mathrm{dp})\end{array}$ \\
\hline Homens & 415 & $44,42(8,21)$ & $16,05(3,61)$ & $12,90(3,56)$ & $15,52(3,63)$ \\
\hline Mulheres & 253 & $43,44(8,87)$ & $15,82(3,75)$ & $12,79(4,15)$ & $15,34(3,70)$ \\
\hline Total & 668 & $44,21(8,50)$ & $15,95(3,67)$ & $12,85(3,83)$ & $15,44(3,66)$ \\
\hline
\end{tabular}

A análise de variância não revelou diferenças significativas entre os trabalhadores celetistas e estatutários no escore total de comprometimento, $\underline{F}(1,596)=1,98, p=0,16$, e nos escores parciais de identidade, $\underline{\mathrm{F}}(1,596)=0,87, p=0,77$, e resiliência, $\underline{\mathrm{F}}(1,596)=1,22, p=0,27$. Foi encontrada diferença significativa nos escores de planejamento, $\underline{F}(1,596)=5,19, p=$ 0,02 . Os trabalhadores celetistas revelaram escores mais elevados de planejamento de carreira em comparação com os trabalhadores em regime estatutário. A Tabela 5 apresenta dados de estatística descritiva dos escores de comprometimento em cada tipo de contrato de trabalho. 
Tabela 5. Médias (M), desvios-padrão (dp) e valores de $\mathrm{N}$ para os escores de comprometimento com a carreira na dependência do tipo de contrato de trabalho

\begin{tabular}{lccccc}
\hline $\begin{array}{l}\text { Contrato de } \\
\text { trabalho }\end{array}$ & $\mathrm{N}$ & $\begin{array}{c}\text { Comprometimento } \\
\mathrm{M}(\mathrm{dp})\end{array}$ & $\begin{array}{c}\text { Identidade } \\
\mathrm{M}(\mathrm{dp})\end{array}$ & $\begin{array}{c}\text { Resiliência } \\
\mathrm{M}(\mathrm{dp})\end{array}$ & $\begin{array}{c}\text { Planejamento } \\
\mathrm{M}(\mathrm{dp})\end{array}$ \\
\hline CLT & 387 & $44,98(8,53)$ & $16,16(3,69)$ & $13,02(3,77)$ & $15,83(3,66)$ \\
Estatutário & 211 & $43,97(8,23)$ & $16,25(3,45)$ & $12,66(3,85)$ & $15,11(3,72)$ \\
Total & & $44,62(8,43)$ & $16,19(3,61)$ & $12,90(3,80)$ & $15,58(3,69)$ \\
\hline
\end{tabular}

\section{Discussão}

Os dados colhidos com a Escala de Comprometimento com a Carreira mostraram-se adequados ao procedimento da análise fatorial exploratória. O resultado obtido com o gráfico Scree confirmou a estrutura dimensional original de Carson e Bedeian (1994), recomendando a solução fatorial dos itens a três fatores. Nesse sentido, três fatores obtiveram autovalores adequados e explicaram mais da metade da variância dos escores de comprometimento, portanto, a solução fatorial mais adequada parece ser a trifatorial.

Além de os três fatores explicarem uma parte importante da variância total, todos os itens da ECC saturaram nas dimensões para as quais foram elaborados. Embora um item tenha saturado acima de 0,30 em mais de um fator, a saturação mais elevada ocorreu no fator definido pelo modelo. Os demais itens mostraram saturação acima de 0,30 somente nos fatores teoricamente esperados, de forma a permitir que a validade de construto (via análise fatorial exploratória) seja assumida.

Sobre o item com saturação em mais de um fator, este corresponde à frase "eu não costumo pensar sobre o meu desenvolvimento pessoal nesta linha de trabalho/campo de carreira". Esse item saturou nos fatores correspondentes às dimensões identidade e planejamento de carreira. Essa sobreposição pode ser compreendida com a análise da sua formulação, que não se mostra suficientemente clara na representação da dimensão planejamento. Além disso, observa-se que se trata de uma frase negativa (escores devem ser invertidos), característica que pode gerar dificuldades para o respondente. Embora essa seja a tradução fiel à versão original do instrumento, sugere-se que os próximos estudos brasileiros explorem novas formulações desse item ou novos itens para a dimensão planejamento, tais como "eu costumo pensar sobre o meu futuro nesta linha de trabalho/campo de carreira".

A avaliação dos índices de consistência interna da escala total e de cada dimensão de comprometimento revelou resultados positivos, com níveis de precisão totais e parciais satisfatórios. Todos os itens contribuíram para a precisão da sua respectiva dimensão, indicando a relevância da sua permanência no instrumento.

Não ocorreram diferenças de comprometimento associadas ao nível de escolaridade entre os escores totais de comprometimento e entre os escores parciais das dimensões de planejamento e de resiliência. Na dimensão identidade de carreira, trabalhadores com escolaridade em nível de pós-graduação apresentaram escores mais elevados do que aqueles com escolaridade de ensino técnico. 
Pode-se pensar que as características de instabilidade e de incerteza que cercam as condições de trabalho e emprego na atualidade pressionem por comportamentos de planejamento e resiliência em trabalhadores de todos os níveis de escolaridade. Se a estabilidade do mundo do trabalho em tempos passados não exigia tais comportamentos em alto grau, o cenário emergente demanda de todos os trabalhadores o incremento da iniciativa e do planejamento independentemente da vida e da carreira. Por outro lado, a identidade de carreira, que corresponde à importância pessoal de determinado campo de trabalho, pode adquirir magnitudes diversas na dependência das possibilidades socioeconômicas individuais para a dedicação a áreas de carreira de maior interesse pessoal, tal como geralmente ocorre na escolha e na realização de cursos de pós-graduação. Nesse sentido, vale acrescentar que a percepção de adequação pessoal ao trabalho é um dos preditores mais fortes de comprometimento com a carreira (Carless, 2005).

Em relação ao tipo de contrato de trabalho, os dados confirmaram a expectativa que trabalhadores em contratos celetistas, sem as garantias de estabilidade no emprego associadas ao regime estatutário, se preocupem mais com o futuro de suas carreiras e com o planejamento necessário à manutenção de sua empregabilidade.

As comparações de gênero não indicaram diferenças significativas entre homens e mulheres nos escores totais e parciais de comprometimento com a carreira. Nesse sentido, a pesquisa recente de Lassance (2010) revelou maior flexibilidade dos papéis tradicionais de gênero em termos da importância das atividades de trabalho e familiares para homens e mulheres. Mulheres têm revelado maior conexão com o trabalho, e homens, maior envolvimento em atividades familiares, de modo a equilibrar inclinações tradicionais na direção da maior igualdade entre os sexos (Lassance, 2010; Nomaguchi, 2009).

A Escala de Comprometimento com a Carreira tem sido utilizada em amostras brasileiras e tem revelado associações teoricamente relevantes com outras variáveis, tais como o comprometimento organizacional, o entrincheiramento na carreira, a motivação vital, a generatividade, o comprometimento organizacional e os interesses vocacionais (Baiocchi \& Magalhães, 2004; Magalhães \& Gomes, 2005; Magalhães \& Gomes, 2007; Magalhães, 2008); portanto, sugere-se ser esse um instrumento adequado à realidade brasileira, e recomenda-se o seu uso em pesquisas que relacionem o construto a outros fatores e processos de interesse.

\section{Considerações finais}

Medidas relacionadas ao comportamento no trabalho têm se mostrado particularmente suscetíveis à obsolescência, dadas as transformações constantes que atingem o significado e as relações de trabalho na contemporaneidade. O comprometimento com a carreira está entre os constructos teóricos que sofreram esses impactos. As diferenças apontadas entre as definições e as operacionalizações mais utilizadas do constructo revelaram divergências que expressam as mudanças decorrentes no significado de carreira e nos comportamentos, que passaram a ser percebidos como comprometimento com a carreira. Acreditase que a Escala de Comprometimento com a Carreira de Carson e Bedeian (1994), objeto deste estudo, aproxima-se das definições mais recentes do que seja um trabalhador comprometido com a sua carreira, e sugerese a sua utilização em pesquisas sobre o tema.

O constructo permanece relevante para trabalhadores, gestores e profissionais de recursos humanos. A influência do 
comprometimento com a carreira em diversos processos de interesse para o indivíduo e para a organização, tais como o desenvolvimento de competências e a retenção de empregados (Aryee \& Tan, 1992), a satisfação e o desempenho, tanto em trabalhadores permanentes (Mrayyan \& Al-Faouri, 2008) quanto temporários (Niu, 2011), e o bem-estar no trabalho (Duffy, 2011), recomenda a investigação dos fatores que possam promover ou enfraquecer a experiência de comprometimento nos trabalhadores. Além disso, o construto tem se mostrado efetivo para a pesquisa e o entendimento de temas correlatos, tais como o conflito trabalho-família (Okurami, 2012).

Por fim, sugere-se que pesquisas futuras investiguem os antecedentes e os consequentes dos diversos componentes do comprometimento com a carreira (identidade, resiliência e planejamento), explorando o significado psicológico e a relevância de cada um desses fatores para a compreensão de variáveis e processos em nível individual e organizacional.
Referências

\section{Mauro de Oliveira Magalhães}

Doutor em Psicologia do Desenvolvimento pela Universidade Federal do Rio Grande do Sul e docente do Instituto de Psicologia e da pós-graduação em Psicologia da Universidade Federal da Bahia, Salvador - BA - Brasil. Email: mauro.m@terra.com.br

Endereço para envio de correspondência:

Rua Tenente Pires Ferreira 308, Bloco A, Ap. 201. CEP: 40130-160. Salvador, BA.

Recebido 17/01/2012, 1a Reformulação 23/11/2012, Aprovado 29/11/2012.

Aryee, S., \& Tan, K. (1992). Antecedents and outcomes of career commitment. Journal of Vocational Behavior, 40(3), 288-305.

Baiocchi, A., \& Magalhães, M. (2004). Relações entre processos de comprometimento, comprometimento e motivação vital em carreiras profissionais. Revista Brasileira de Orientação Profissional, 5(1), 63-71

Bastos, A. V. B. (1992). Medidas de comprometimento no contexto de trabalho: um estudo preliminar de validade discriminante. Psico, 24(2), 29-48.

Bastos, A.V. (1997). A escolha e o comprometimento com a carreira: um estudo entre profissionais e estudantes de administração. Revista de Administração, 32(3), 28-39.

Bastos, A. V. B., Magalhães, M. O., \& Carvalho, T. A. (2010). Os vínculos do psicólogo com o seu trabalho: uma análise do comprometimento com a profissão e com a área de atuação. In A. V. B. Bastos, S. M. G. Gondin (Orgs.). O trabalho do psicólogo no Brasil (pp. 303-326). Porto Alegre: Artmed.

Blau, G. J. (1985). The measurement and prediction of career commitment. Journal of Occupational Psychology, 58, 277288.

Blau, G. J. (1988). Further exploring the meaning and measurement of career commitment. Journal of Vocational Behavior, 32, 284-297.

Blau, G. J. (2003). Testing for a four-dimensional structure of occupational commitment. Journal of Occupational and Organizational Psychology, 76, 469-488.

Carless, S. A. (2005). The influence of fit perceptions, equal opportunity policies, and social support network on pre-entry 
police officer career commitment and intentions to remain. Journal of Criminal Justice, 33, 341-352.

Carson, K. D., \& Bedeian, A. G. (1994). Career commitment: Construction of a measure and examination of its psychometric properties. Journal of Vocational Behavior, 44(3), 237-262.

Carson, K. D., Carson, P. P., \& Bedeian, A. G. (1995). Development and construct validation of a career entrenchment measure. Journal of Occupational and Organizational Psychology, 68, 301-320.

Chang, E. (1999). Career commitment as a complex moderator of organizational commitment and turnover intention. Human Relations, 52(10), 1258-1278.

Conselho Nacional de Saúde. (1996). Resolução para pesquisa envolvendo seres humanos. Resolução $n^{\circ}$ 196/1996, Brasília, DF.

Dassa, C. (1999). Analyse multidimensionnelle exploratoire et confirmative. Montreal: Université Montreal.

Duffy, R. (2011). Calling and work-related outcomes: Career commitment as a mediator. Journal of Vocational Behavior, 78 (2), $210-218$.

Fu, J. (2011). Understanding career commitment of IT professionals: Perspective of push-pull-mooring framework and investment model. International Journal of Information Management, 31, 279-293.

Goulet, R., \& Singh, P. (2002). Career commitment: A reexamination and an extension. Journal of Vocational Behavior, 61, 73-91.

Grzeda M. (1999). Career development and emerging managerial career patterns. Journal of Career Development, 25(4), 233-248.

Hughes, E. C. (1958). Men and their work. Glencoe, IL: Free Press.

Lassance, M. C. P. (2010). Saliência de papel, valores de trabalho e tarefas de desenvolvimento de carreira. Tese de doutorado. Universidade Federal do Rio Grande do Sul, Porto Alegre.

London, M. (1983). Toward a theory of career motivation. Academy of Management Review, 8(4), 620-630.

Magalhães, M. O. (2005). Personalidades vocacionais e desenvolvimento na vida adulta: generatividade e carreira profissional. Tese de doutorado. Universidade Federal do Rio Grande do Sul, Porto Alegre.

Magalhães, M. O., \& Gomes, W. B. (2005). Personalidades vocacionais, generatividade e carreira na vida adulta. Revista Brasileira de Orientação Profissional, 6, 71-79.

Magalhães, M. O., \& Gomes, W. B. (2007). Personalidades vocacionais e processos de carreira na vida adulta. Psicologia em Estudo, 12(1), 95-103.

Magalhães, M. O. (2008). Generatividade e vínculos com a carreira e com a organização: problematizando as bases do comprometimento organizacional. Revista Brasileira de Orientação Profissional, 9, 67-80.

Meyer, J., Allen, N., \& Smith, C. (1993). Commitment to organizations and occupations: Extension and test of a threecomponent conceptualization. Journal of Applied Psychology, $78,538-551$.
Meyer, J. P., \& Herscovitch, L. (2001). Commitment in the workplace toward a general model. Human Resource Management Review, 11, 299-326.

Morrow, P. (1993). The theory and measurement of work commitment. Greenwich, CT: JAI.

Mrayyan, M. T., \& Al-Faouri, I. (2008). Predictors of career commitment and job performance of Jordanian nurses. Journal of Nursing Management, 16, 246-256.

Niu, Han-Jen. (2011). The career commitment of contingent employees: Evidence of on-site engineers in semiconductor industry. International Management Review, 7(2), 44-51.

Nomaguchi, K. M. (2009). Change in work-family conflict among employed parents between 1977 and 1997. Journal of Marriage and Family, 71, 15-32.

Okurame, D. (2012). Linking work family conflict to career commitment: The moderating effect of gender and mentoring among Nigerian civil servants. Journal of career development, 39(5), 423-442.

Riveros, A. M. M., \& Tsai, T. S. (2011). Career commitment and organizational commitment in for-profit and non-profit sectors. International Journal of Emerging Sciences, 1(3), 324-340.

Rodrigues, A. C., \& Bastos, A. V. B. (2010). Problemas conceituais e empíricos na pesquisa sobre comprometimento organizacional: uma análise crítica do modelo tridimensional de J. Meyer e N. Allen. Psicologia: Organizações e Trabalho, 10, 129-144.

Rowe, D. E. O., \& Bastos, A. V. B. (2010). Vínculo com a carreira e produção acadêmica: comparando docentes de ies públicas e privadas. Revista de Administração Contemporânea, 14, 1011-1030.

Rowe, D. E. O., \& Bastos, A. V. B. (2011). Comprometimento e entrincheiramento na carreira: um estudo de suas influências no esforço instrucional do docente do ensino superior. Revista de Administração Contemporânea, 15, 973-992.

Schein, E. (1990). Career stress in changing times: Some final observations. Prevention in Human Services, 8(1), 251-261.

Schnell, T. (2011). Individual differences in meaning-making: Considering the variety of sources of meaning, their density and diversity. Personality and Individual Differences, 51(5), 667-673.

Solinger, O. N., Olffen, W., \& Roe, R. A. (2008). Beyond the threecomponent model of organizational commitment. Journal of Applied Psychology, 93(1), 70-83.

Sullivan, S. E., \& Baruch, Y. (2009). Advances in career theory and research: A critical review and agenda for future exploration. Journal of Management, 35, 1542-1571.

Super, D. E., Savickas, M. L., \& Super, C. (1996). Career choice and development. In D. Brown \& L. Brooks (Eds.). The life-span, life-space approach to careers (pp. 121-178). San Francisco: Jossey-Bass Publishers. 